\title{
Molecular characterisation and expression analysis of SEREX-defined antigen NUCB2 in gastric epithelium, gastritis and gastric cancer
}

\author{
Z. Kalnina, ${ }^{1}$ K. Silina, ${ }^{1}$ R. Bruvere, ${ }^{1}$ N. Gabruseva, ${ }^{1}$ A. Stengrevics, ${ }^{2}$ S. Barnikol-Watanabe, ${ }^{3}$ \\ M. Leja, ${ }^{4}$ A. Line ${ }^{1}$ \\ ${ }^{1}$ Latvian Biomedical Research and Study Centre, Riga, Latvia; ' Latvian Oncology Centre, Riga, Latvia; \\ ${ }^{3}$ Department of Immunochemistry, Max Planck Institute for Experimental Medicine, Göttingen, Germany; \\ ${ }^{4}$ Faculty of Medicine, University of Latvia, Riga, Latvia
}

(C2009 European Journal of Histochemistry

NUCB2 is an EF-hand $\mathrm{Ca}^{2+}$ binding protein that has been implicated in various physiological processes like calcium homeostasis, hypothalamic regulation of feeding and TNF receptor shedding. In our previous study we identified NUCB2 as a potential tumour antigen eliciting autoantibody responses in $5.4 \%$ of gastric cancer patients but not in the healthy individuals. The current study aimed to elucidate the molecular mechanism underlying NUCB2 immunogenicity and to gain an insight into the physiological functions of NUCB2 in the stomach. mRNA expression analysis demonstrated that NUCB2 is ubiquitously expressed in normal tissues, including lymphoid tissues, and downregulated in gastric tumours when compared with the adjacent relatively normal stomach tissues. The search for molecular alterations resulted in the identification of novel mRNA variants transcribed from an alternative promoter and expressed predominantly in gastric cancers. Western blot analysis demonstrated that the protein levels correspond to mRNA levels and revealed that NUCB2 is phosphorylated in gastric mucosa. Furthermore, a $55 \mathrm{kDa}$ isoform, generated presumably by yet an unidentified post-translational modification was detected in gastric tumours and AGS gastric cancer cells but was absent in the relatively normal gastric mucosa and thereby might have served as a trigger for the immune response against NUCB2. Staining of stomach tissue microarray with anti-NUCB2 antibody revealed that it is expressed in the secretory granules of chief cells and in the cytoplasm of parietal cells in the functioning gastric glands which are lost in atrophic glands and tumour cells. Hence we propose that NUCB2 may be implicated in gastric secretion by establishing an agonist-releasable $\mathrm{Ca}^{2+}$ store in ER or Golgi apparatus, signalling via heterotrimeric $\mathrm{G}_{\alpha}$ proteins and/or mediating the exocytosis of the secretory granules.

Key words: NUCB2, SEREX, tumour-associated antigens, chief cells, parietal cells, gastric cancer, pepsinogen secretion.

Correspondence: Zane Kalnina,

Latvian Biomedical Research and Study Centre, 1

Ratsupites St, LV-1067, Riga, Latvia

Tel.: 00371.67808208.

Fax: 00371.67442407

E-mail: zane@biomed.Iu.Iv

Paper accepted on October 24, 2008

European Journal of Histochemistry

2009; vol. 53 issue 1 (January-March): 7-18
$\mathrm{N}$ ucleobindin 2 (NUCB2 or NEFA) is a ubiquitously expressed EF-hand $\mathrm{Ca}^{2+}$ binding protein that may be implicated in various physiological processes, however, its functional role and mechanism of action are poorly understood. So far, two nucleobindins - NUCBI (or Calnuc, Nuc) and NUCB2 have been identified in humans (Wendel et al. 1995; Barnikol-Watanabe et al. 1994). They share $61.65 \%$ amino acid identity and a characteristic composition of functional domains - a signal peptide, a Leu/Ile rich region, basic amino acid rich region including putative bipartite nuclear localisation signal, two $\mathrm{Ca}^{2+}$ binding EF-hand domains separated by acidic amino acid rich region and a leucine zipper (Figure 1) (Barnikol-Watanabe et al. 1994; Miura et al. 1992). Genes encoding NUCB1 and NUCB2 are located in 19q13.2-q13.4 and 11p15.1-p14, respectively, and are deemed to be derived from a common four-domain EF-hand ancestor (Karabinos et al. 1996). Within the cell both are located in the cytoplasmic and Golgi luminal pools, both are secreted into extracellular space and interact with other proteins in a calciumdependent manner (Lavoie et al. 2002; Lin et al. 2000; Morel-Huaux et al. 2002; Taniguchi et al. 2000; Islam et al. 2006). Both nucleobindins were reported to interact with the postmitotic growth suppressor Necdin in neuronal cytoplasm (Taniguchi et al. 2000) and have been shown to constitute a $\mathrm{Ca}^{2+}$ storage pool in Golgi ( $\mathrm{Lin}$ et al. 1999; Kroll et al. 1999; Morel-Huaux et al. 2002). NUCBI interacts with cyclooxygenases in COS-1 cells (Ballif et al. 1996) and with G protein Gai3 in the cytoplasm of rat kidney cells (Lin et al. 2000), and acts as a negative regulator in ATF6-mediated unfolded protein response (Tsukumo et al. 2007). It is secreted via constitutive secretory pathway in AtT20 pituitary cells (Lavoie et al. 2002). NUCB2 has been shown to 
form a calcium-dependent complex with ARTS-1 that mediates release of TNF receptor to the extracellular compartment from human vascular endothelial cells (Islam et al. 2006). Furthermore, nesfatin- 1 - the $N$ terminal 82 amino acid peptide generated by post-translational processing of NUCB2 in rat hypothalamus - was recently shown to be involved in feeding regulation and suggested to suppress appetite via leptin-independent signalling (Oh et al. 2006) and is upregulated by serotonin receptor agonists (Nonogaki et al. 2008).

In a previous study we identified NUCB2 as an antigen eliciting autoantibody production in gastric cancer patients (Line et al. 2002) by exploiting the SEREX (serological identification of antigens by recombinant expression cloning) approach (Sahin et al. 1997). The screening of gastric cancer-derived cDNA expression library with allogeneic serum resulted in the identification of 5 different serum-reactive phage clones, including Zg4 encoding the 31-179 aa region of NUCB2 (Figure 1). Anti-NUCB2 antibodies were found exclusively in patients with gastric cancer and not in colon and breast cancer patients or healthy donors. No immune responses against NUCB2 have been reported so far, while NUCBI has been shown to be overexpressed in $59.4 \%$ of colon cancer specimens and elicit autoantibody responses in $11.5 \%$ of the cases (Chen et al. 2007). Furthermore, Nucbl is excessively produced in lupus-prone mice and induces autoantibody response against itself and a number of other lupus-associated autoantigens when injected in normal BALB/c mice, and the antibody production is mediated by autoreactive CD4 ${ }^{+} \mathrm{T}$ cells (Kanai et al. 1995; Kubota et al. 2001).

The aim of the current study is to identify the putative molecular alterations underlying the immunogenicity of NUCB2 in gastric cancer patients and to get an insight into its physiological role in gastric mucosa.

\section{Materials and Methods}

\section{Tissue and serum samples and cell line}

Gastric cancer and adjacent relatively normal tissue samples from 36 patients (Table 1) undergoing surgery at the Latvian Oncology Centre were collected intraoperatively and one part of each specimen was immediately submerged in RNAlater (Ambion) and subsequently used for RNA extraction while another was embedded in paraffin blocks. Sera were collected from the same patients whose tissue specimens were collected and from another 20 gastric cancer patients, 23 colorectal and 11 breast cancer patients and 13 patients with chronic atrophic gastritis at the time of diagnosis. Sera from 36 healthy individuals were obtained from the Genome Database of the Latvian population. This study was approved by the Central Committee of Medical Ethics of Latvia and the tissue samples and sera were collected after the patients' informed consent was obtained.

AGS gastric cancer cell line was purchased from ECACC and grown in monolayer culture in Ham's F12 supplemented with $2 \mathrm{mM}$ glutamine and $10 \%$ FBS (Invitrogen).

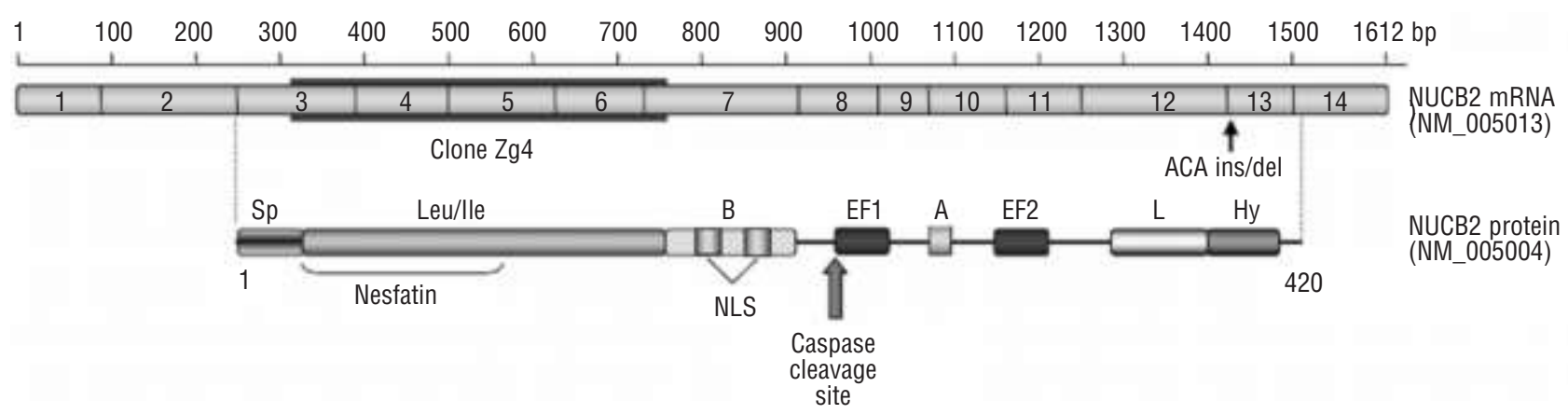

\footnotetext{
Figure 1. The schematic representation of NUCB2 exon composition and the functional domains of the protein. Sp, signal peptide; Leu/Ile, Leu and Ile rich region; B, basic amino acid rich region; NLS, putative bipartite nuclear localisation signal; EF1, EF2, Ca ${ }^{2+}$ binding EF-hand domains; A, acidic amino acid rich region; L, leucine zipper; Hy, hydrophobic region.
} 
Table 1. Clinicopathological characteristics of the gastric cancer patients.

\begin{tabular}{ll}
\hline Variables & Number (\%) \\
\hline Total & $36(100 \%)$ \\
Sex & \\
$\quad$ Male & $15(41.7)$ \\
Female & $21(58.3)$ \\
Age (years) & \\
$\quad$ Mean (range) & $66(34-85)$ \\
Tumour location & \\
Upper third & $1(2.8)$ \\
Middle third & $18(50)$ \\
Lower third & $13(36)$ \\
Total & $1(2.8)$ \\
Not determined & $3(8.4)$ \\
Grade & \\
G1 & $1(2.8)$ \\
G2 & $6(16.7)$ \\
G3 & $21(58.3)$ \\
G4 & $5(14)$ \\
Not determined & $3(8.4)$ \\
Tumour stage & \\
IA & $5(14)$ \\
IB & $1(2.8)$ \\
II & $12(33.3)$ \\
IIIA & $14(39)$ \\
IV & $2(5.6)$ \\
Not determined & $2(5.6)$ \\
\hline
\end{tabular}

\section{Serological analysis}

The frequency of anti-NUCB2 antibody responses in allogeneic sera was determined by plaque assay as described previously (Line et al. 2002). Briefly, E. coli XL1 blue cells were transfected with the lambda phages by spotting 20-30 pfu of Zg4 phage side by side with non-recombinant phage directly on an agar plate and the phage arrays were screened with 1:200 diluted sera, which have been previously preabsorbed with $E$. coli-phage lysates.

The presence of antibodies against gastric parietal cell (GPC) $\mathrm{H}+/ \mathrm{K}+$ ATPase antigen was tested semi-quantitatively in the sera from 13 atrophic gastritis patients and 16 gastric cancer patients by enzyme linked immunosorbent assay exploiting AUTOSTATTM Anti-Gastric Parietal Cell kit (Hycor Biomedical) following manufacturer's instructions.

\section{Real-time RT-PCR analysis}

NUCB2 mRNA expression pattern was analysed in a panel of normal tissues consisting of whole bone marrow, brain, colon, heart, kidney, lung, pancreas, skin, spleen, stomach, testis, thymus and trachea (BioCat, Ambion) and compared between gastric cancer and adjacent non-cancerous tissues by real-time RT-PCR. Total RNA was isolated from RNAlater preserved cancerous and adjacent normal tissue specimens using TRI reagent (Sigma) according to the manufacturer's instructions. Four micrograms of total RNA were used to synthesise first-strand cDNA by random hexamer priming using First-Strand cDNA Synthesis Kit (MBI Fermentas). To choose the appropriate reference genes for each tissue panel, a set of seven candidate reference genes (TBP, POLR2A, YWHAZ, ACTB, GAPDH, PGK1, TUB3A) were tested for their mRNA expression stability in the given panel and three most stable genes (TUB3A, ACTB and POLR2A for normal tissue panel and ACTB, TBP and POLR2A for gastric cancer and the adjacent tissue panel) were determined by using the openaccess software GeNorm (http://medgen.ugent.be/ $\sim$ jvdesomp/genorm). These genes were used to calculate the factor which was applied for the normalisation of the obtained NUCB2 mRNA expression data. The following gene-specific primers were used for the amplification of NUCB2 and the reference genes: NUCB2 forward 5'-TCAAGCAAGTGATTGATGTGC-3', reverse 5'-TCAGGATTCA GGTGGTTTAGG-3'; ACTB forward 5'-AGTGTGACGTGGACATCCG-3', reverse 5'-AATCTCATC TTGTTTTCTGCGC-3; TUB3A forward 5'-TATGGCAAGAAGTCCAAGCTG-3', reverse 5'-TACCATGAAGGCACAATCAGAG-3'; POLR2A forward 5'GGGTCATCTTCCCAACTGGAG-3', reverse 5'CACCAGCTTCTTGCTCAATTCC- $3^{\prime}$ and TBP forward 5'-CCACTCACAGACTCTCACAAC-3', reverse 5'-CTGCGGTACAATCCCAGAAC-3'. In all experiments $1 / 30$ of each RT mixture was amplified in a total reaction volume of $20 \mu \mathrm{L}$ containing 2 pmol of each primer and $10 \mu \mathrm{L}$ SYBR Green mix (ABgene) exploiting 7500 Real Time PCR System (Applied Biosystems). All reactions were performed in duplicates.

\section{5' RLM-RACE and RT-PCR analysis}

Full-length $5^{\prime}$ end sequences of NUCB2 CDNA were cloned from 3 gastric cancer specimens using FirstChoice $^{\mathrm{TM}}$ RLM-RACE kit (Ambion) as recommended by the manufacturer. Briefly, $10 \mu \mathrm{g}$ of total RNA underwent $5^{\prime}$ phosphate removal from uncapped RNAs by Calf Intestinal Phosphatase treatment and next, cap structures from full-length mRNAs were removed with Tobacco Acid Pyrophosphatase leaving a free $5^{\prime}$ phosphate. mRNA molecules containing $5^{\prime}$ phosphate were 
subjected to the ligation of RNA adapters to their $5^{\prime}$ ends. Random-primed reverse transcription was performed with subsequent nested PCR using NUCB2-specific reverse primers (5'-TCAGGATT CAGGTGGTTTAGG-3' for PCR 1 and 5'-CTATGTCTGCTTTCTGGAGC-3' for PCR 2) and adapter-specific forward primers. PCR products were cloned using InsTAclone ${ }^{\mathrm{TM}} \mathrm{PCR}$ Product cloning Kit (MBI Fermentas) and multiple clones were sequenced. The expression of the identified NUCB2 transcript variants in normal tissues (brain, liver, heart, kidney, lung, trachea, spleen, colon, stomach, testis and ovary) (Ambion) and paired gastric cancer and adjacent normal tissues was analysed by RT-PCR using alternative forward primers located in exon 1 5'-CAGGTTTGTGCGCTGGACG-3' and in exon la 5'-AGAAATCTCCTGGAATCAAGG-3' and common reverse primer 5'-CTATGTCTGCTTTCTGGAGC-3'. GAPDH was used as the endogenous control.cDNA synthesis was carried out as described above and 1/15 of RT mixture was amplified in a total reaction volume of 20 $\mu \mathrm{L}$ containing $10 \mathrm{pmol}$ of each primer, $200 \mu \mathrm{M}$ dNTPs and $2 \mathrm{U}$ of Taq polymerase (MBI Fermentas) in the following cycling conditions: 1 min at $94^{\circ} \mathrm{C}, 30 \mathrm{~s}$ at $58^{\circ} \mathrm{C}$ and $45 \mathrm{~s}$ at $72^{\circ} \mathrm{C}$. Twenty five cycles of amplification were performed for GAPDH, 27 for NUCB2 transcripts containing exon 1 and 35 for those containing exon 1 a.

\section{Western blot analysis}

NUCB2 protein levels in gastric cancer, adjacent normal tissues and AGS cell line were analysed by Western blotting. Total protein was isolated from tissue samples using TRI reagent (Sigma) simultaneously with RNA according to manufacturer's instructions. Ten $\mu \mathrm{g}$ of total protein were resuspended in Laemmli sample buffer, denatured for 5 min at $100^{\circ} \mathrm{C}$ and separated by $10 \%$ SDS-PAGE. Proteins were electroblotted onto Protan nitrocellulose membrane (Whatman) and stained with Ponceau S solution to verify equal protein loading. The membrane was destained, blocked with $5 \%(\mathrm{w} / \mathrm{v})$ fatfree milk in TBS and probed with 1:200 diluted mouse anti-NUCB2 monoclonal antibody, kindly provided by Dr. Shitsu Barnikol-Watanabe (Gottingen, Germany). Antigen-antibody complexes were detected using HRP-conjugated goat antimouse secondary antibody (Sigma) (diluted 1:5000 in TBS) and ECL detection system (GE Healthcare) according to the manufacturer's instructions. In parallel, secondary antibody alone was used for probing to provide the negative control.

\section{Dephosphorylation and deglycosylation experiments}

On-line open access softwares (http://www. cbs.dtu.dk/services and http://www.expasy.ch/tools) were used for the prediction of protein phosphorylation and glycosylation sites. To assess the phosphorylation status, protein samples were treated with $1 \mathrm{U}$ of SAP (MBI Fermentas) for $1 \mathrm{~h}$ at $37^{\circ} \mathrm{C}$ and then subjected to Western blot analysis as described above. Deglycosylation was carried out using Enzymatic deglycosylation kit (Glyko) according to the manufacturer's instructions. The protein extracts were denatured by heating at $100^{\circ} \mathrm{C}$ for $5 \mathrm{~min}$ in SDS and $\beta$-mercaptoethanol and then incubated with $\mathrm{N}$-Glycanase $(5 \mathrm{U} / \mathrm{mL})$, Sialidase A $(5 \mathrm{U} / \mathrm{mL}), 0$-Glycanase $(1.25 \mathrm{U} / \mathrm{mL})$, $\beta$ (1-4)-Galactosidase (2 U/mL) and $\beta$-N-Acetylglucosaminidase $(40 \mathrm{U} / \mathrm{mL})$ for 3 hours at $37^{\circ} \mathrm{C}$ and analysed by Western blot. Bovine Fetuin was treated in parallel as a positive control.

\section{Immunohistochemistry}

To investigate the NUCB2 expression in different regions of stomach and various gastric lesions, human tissue array containing a total of 63 cores from multiple sites in normal stomach, and a total of 80 cores from superficial gastritis, mild, moderate and severe chronic atrophic gastritis with intestinal metaplasia as well as grade I-III adenocarcinoma was purchased from Cybrdi and processed using mouse anti-NUCB2 monoclonal antibody (at $1: 20$ dilution in PBS, 1\% BSA) kindly provided by Dr. Shitsu Barnikol-Watanabe (Gottingen, Germany) and Mouse UniTec ABC kit (Calbiochem) according to the manufacturer's instructions. To study NUCB2 protein expression in gastric cancers, $4 \mu \mathrm{m}$ thick sections were prepared from formalin-fixed, paraffin-embedded gastric cancer and adjacent normal tissue specimens from the same patients whose tissues were used for RTPCR and Western blot analyses and mounted on poly-L-lysine-coated slides, deparaffinised in xylene and rehydrated by placing in 100\%, 95\%, $80 \%, 70 \%, 50 \%$ ethanol and PBS. Antigen retrieval was performed by boiling in microwave in $10 \mathrm{mM}$ sodium citrate buffer for $15 \mathrm{~min}$. Next, the sections were washed in PBS and the endogenous peroxidases were quenched with $0.3 \%$ hydrogen peroxide in methanol for $30 \mathrm{~min}$ at RT. Then the 
sections were blocked with 5\% BSA in PBS, $0.5 \%$ Tween-20 for $1 \mathrm{~h}$ and incubated with mouse antiNUCB2 monoclonal antibody, at 1:20 dilution in PBS, $1 \%$ BSA overnight at $4{ }^{\circ} \mathrm{C}$. Slides were washed in PBS, $0.1 \%$ Tween-20 and then incubated with HRP-conjugated goat anti-mouse secondary antibody (Sigma) at 1:100 dilution for $1 \mathrm{~h}$ at RT. To visualise antigen-antibody complexes, sections were stained for 10 min with DAB solution (Sigma) and counterstained with Mayer's haematoxylin. Sections were dehydrated in $70 \%, 96 \%$ and $100 \%$ ethanol for 3 min each, cleared in carboxylol and covered with mounting balsam and cover slips. In parallel, negative controls were performed by substituting primary antibody with nonimmunised mouse serum.

\section{Results}

\section{Humoural responses to NUCB2 are gastric cancer- specific}

The frequency of autoantibodies against NUCB2 was determined by plaque assay in the sera from healthy individuals $(n=36)$ and patients of gastric cancer $(n=56)$, colorectal cancer $(n=23)$, breast cancer $(n=11)$ and chronic atrophic gastritis $(n=13)$. Anti-NUCB2 antibodies were found in 3 gastric cancer patients $(5.36 \%)$, including the patient whose serum was used for searching of tumour antigens by allogeneic SEREX screening but not in the healthy individuals and patients with colorectal or breast cancer or gastritis ( $\chi^{2}$ test: $p=0.158$ relative to healthy donors).

\section{NUCB2 $m R N A$ is differentially expressed in normal tissues and downregulated in gastric cancer}

NUCB2 mRNA expression pattern was examined by real-time RT-PCR in a panel of RNAs derived from various normal tissues and gastric cancer and adjacent normal tissues (Table 1 ). This demonstrated that NUCB2 mRNA is predominantly expressed in pancreas, while its level is 2-3 fold lower in spleen, thymus, bone marrow, testis and stomach and more than 8 fold lower in the remaining normal tissues analysed (Figure $2 \mathrm{~A}$ ). The comparison of mRNA levels between paired gastric cancer and relatively normal tissues showed that it is 2-95 fold downregulated in 28 out of 36 cases analysed (Figure 2 B). The difference between the mean expression values in cancers and normal tissues was statistically significant $(0.35 \pm 0.23$ vs $2.15 \pm 2.04$, paired t-test $p=0.045$ ). No correlation between the expression level and tumour location, grade or stage was observed.

\section{5' RLM-RACE analysis reveal novel NUCB2 tran- script variants}

To search for alternative transcription and translation initiation sites and splice variants of NUCB2, $5^{\prime}$ RLM-RACE analysis was performed resulting in the identification of three novel transcript variants of NUCB2 differing in the exon composition at the $5^{\prime}$ end of the mRNA (transcript variants $b, c$ and $d$ in Figure $3 \mathrm{~A}$ ) (deposited in GenBank, accession numbers EU039831, EU039832 and EU039833). Identification of an alternative first 134 bp exon (1a) located 7408 bp upstream from the conventional first exon suggests the existence of a distal NUCB2 promoter. The other transcript variants differ in an alternative $5^{\prime}$ splice site of the exon 1 that is elongated by $20 \mathrm{bp}$, and an insertion of alternative exon $2 \mathrm{a}$ between exons 2 and 3 . Although alternative exons $1 \mathrm{a}$ and $2 \mathrm{a}$ introduce two independent upstream translation initiation sites, they are followed by coterminous stop codons and therefore
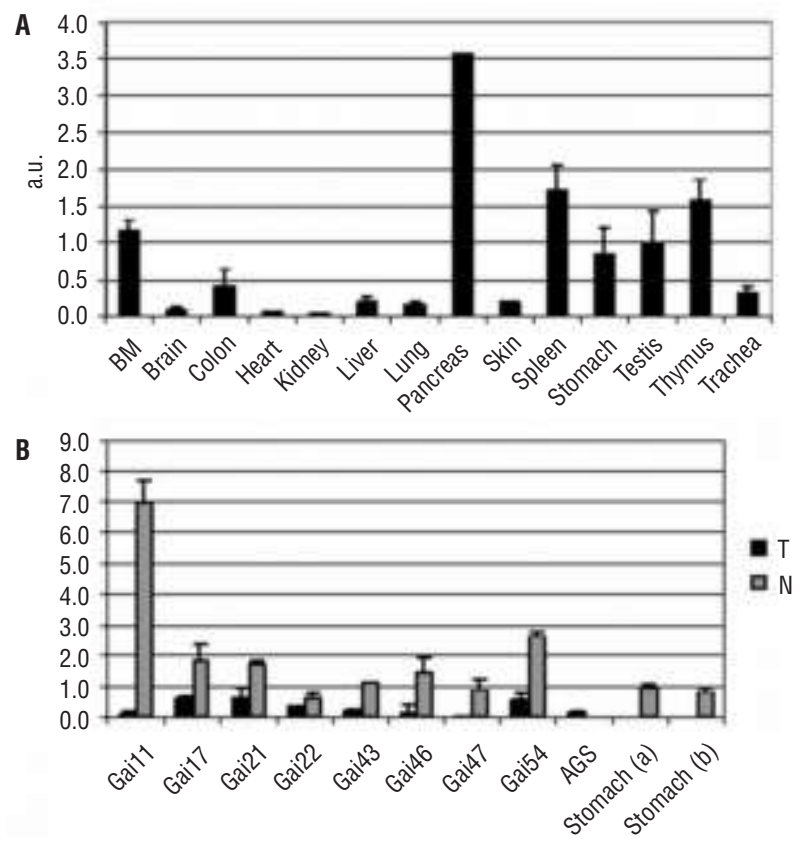

Figure 2. The real-time RT-PCR analysis of NUCB2 mRNA expression in (A) a panel of normal tissue RNAs and (B) paired gastric cancer ( $T$, only selected cases shown) and adjacent relatively normal tissues (N). BM, bone marrow. 
can not give rise to alternative protein isoforms (Figure $3 \mathrm{~A}$ ).

The expression of the NUCB2 transcript variants $\mathrm{a}$ and $\mathrm{b}$ representing proximal and distal promoters, respectively, was examined by RT-PCR in various normal tissues and gastric cancer and adjacent normal tissues from seven patients. This demonstrated that in the normal tissues the distal promoter is used mainly in testis and ovary (Figure 3 B). However, as its detection required 35 cycles of amplification while NUCB2-a was detectable after 27 cycles, it likely is a low-abundance transcript variant. This is also consistent with the results of
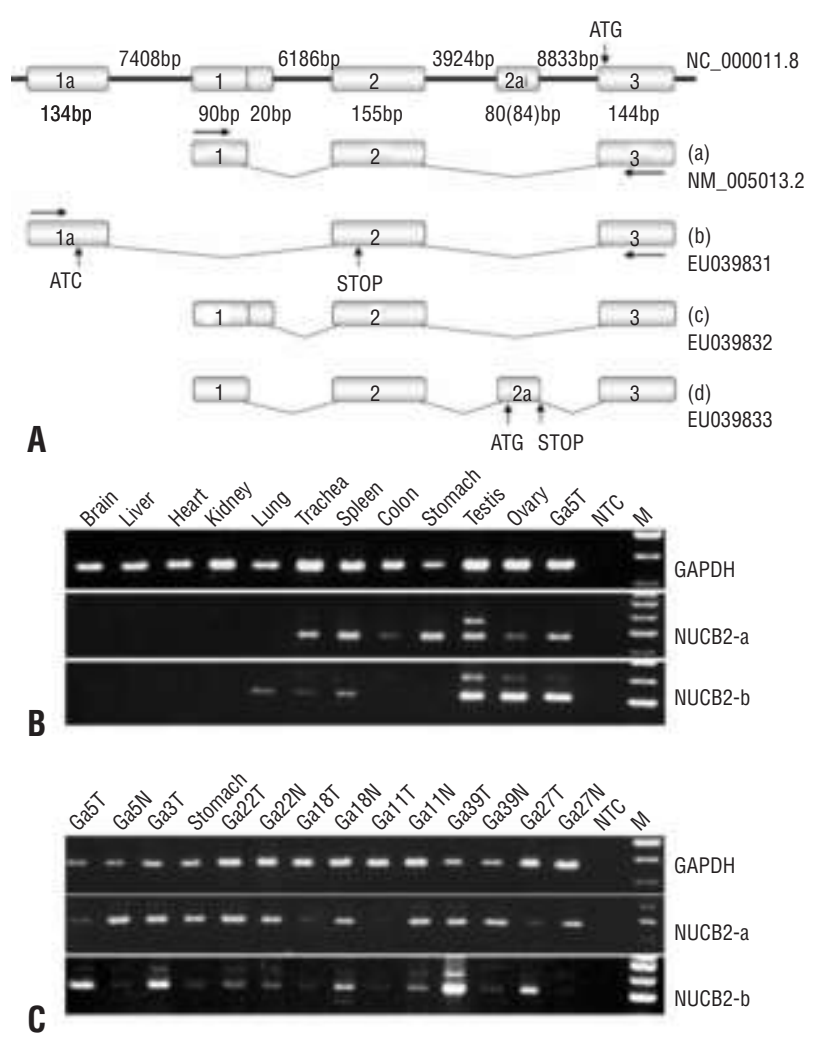

Figure 3. The identification of novel NUCB2 transcript variants by 5'RLM-RACE. (A) The schematic representation of exon-intron composition at the 5' end of NUCB2 gene (NC_000011.8), NUCB2 mRNA reference sequence (NM_005013.2) and newly identified transcript variants (b-c, GenBank accession nos. EU039831-EU039833). The proximal promoter is located upstream of exon 1, the newly identified distal promoter is located upstream of exon 1a. The horizontal arrows indicate the sites of primers used in the expression analysis. The analysis of promoter usage in various normal tissues (B) and paired gastric cancer and adjacent normal tissues (C) by RT-PCR. NUCB2-a represents proximal promoter found in the reference sequence, NUCB2-b - alternative distal promoter. The upper band detected in testis, ovary and Ga39T tissues represents the transcript variant containing exon 2a. 27 and 35 PCR cycles were preformed for amplification of transcript variants a and $b$, respectively. GAPDH was amplified as the internal control using 25 cycles of amplification.
Northern blot revealing a single NUCB2-specific band (data not shown). Usage of the distal promoter was also detected in more than a half of gastric tumours analysed while it was low or undetectable in the adjacent normal tissues (Figure $3 \mathrm{C}$ ). Interestingly, with a single exception, the expression of NUCB2-b transcripts was higher in the tumours with markedly reduced NUCB2-a expression.

\section{Western blot analysis reveal posttranslational modifications of NUCB2}

In order to determine whether the NUCB2 protein level corresponds to its mRNA level, protein extracts of 16 paired gastric cancer and adjacent normal tissues were analysed by Western blot. This showed that the mRNA level correlated with the protein level in all cases analysed and confirmed the downregulation of NUCB2 in cancerous tissues (Figure $4 \mathrm{~A}$ ). It also revealed the presence of additional 52, 53, 55 and $57 \mathrm{kDa}$ species of NUCB2, furthermore 55 and $57 \mathrm{kDa}$ bands were present in 9 out of 16 tumour tissue specimens, including Ga3T - tumour tissue from the patient whose serum was used to search for antigens by SEREX, and AGS gastric cancer cells but not in the relatively normal stomach tissues. Since no putative protein isoforms generated by alternative splicing were identified, we concluded that these bands likely represent post-translationally processed forms of NUCB2. To identify the nature of these modifications, NUCB2 sequence was analysed using tools for the prediction of post-translational modifica-

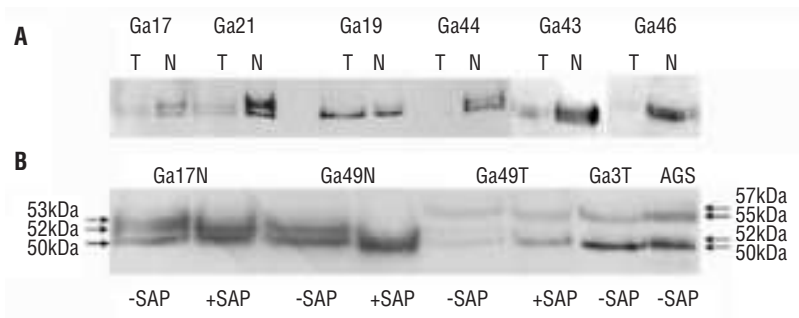

Figure 4. Western blot analyses of NUCB2 expression. (A) NUCB2 was downregulated in the majority of gastric cancer specimens in comparison with adjacent normal tissue and the protein levels correlated with the mRNA levels in all cases. Two to $\mathbf{4}$ closely spaced bands that migrate in the range between $\mathbf{5 0}$ and $57 \mathrm{kDa}$ were detected in gastric tissue specimens (molecular weight of the mature NUCB2 is $\sim 50 \mathrm{kDa})$. (B) The treatment of protein extracts with shrimp alkaline phosphatase (SAP) revealed that 53 and 52 kDa bands in normal tissues and 52 and $57 \mathrm{kDa}$ bands in tumour tissue represented phosphorylated forms of NUCB2. 55 and $57 \mathrm{kDa}$ bands were present only in the tumour tissues, including Ga3T - tumour tissue from the patient with anti-NUCB2 autoantibodies and AGS gastric cancer cells. 
tions at www.expasy.ch/tools/ and www.cbs.dtu.dk/ services. NetPhos 2.0 Server revealed a number of putative Ser, Thr and Tyr phosphorylation sites. To examine the phosphorylation status of NUCB2, the protein extracts were treated with shrimp alkaline phosphatase and subjected to Western blot analysis (Figure 4 B). The treatment removed 53 and 52 $\mathrm{kDa}$ bands in normal tissues and 52 and $57 \mathrm{kDa}$ bands in tumour tissue thus showing that these bands represented phosphorylated forms of NUCB2. However, $52 \mathrm{kDa}$ band in some normal tissue specimens and $55 \mathrm{kDa}$ band in tumours was not affected by the treatment with phosphatase suggesting that they were derived from an alternative post-translational modification. No putative N-glycosylation, acethylation, myristoylation or palmitoylation sites were found by sequence analysis. Although NUCB1 has been shown to undergo 0glycosylation (Lavoie et al. 2002) and 31 Ser and Thr residues were found in NUCB2 sequence, none of them were predicted as potential glycosylation sites by NetOGlyc 3.1 Server. To examine the glyco- sylation status of NUCB2, the protein extracts were treated with Sialidase A and 0-Glycanase but the treatment did not result in the shift of mobility (results not shown). To remove more complex 0linked structures containing $\beta(1-4)$-linked galactose or $\beta$-linked $\mathrm{N}$-acetylglucosamine, the extracts were treated with $\beta$ (1-4)-Galactosidase and $\beta-\mathrm{N}$ Acetylglucosaminidase prior to the 0-glycanase treatment, yet this also did not result in the increase in mobility of NUCB2 bands.

\section{Localisation of NUCB2 in normal gastric epitheli- um and gastric lesions}

To determine which cell types express NUCB2 in normal gastric epithelium and various gastric lesions, a gastric tissue microarray was stained with the anti-NUCB2 monoclonal antibody. This demonstrated that NUCB2 is predominantly localised in the secretory vesicles of chief cells and at lower levels in the cytoplasm of parietal cells in the stomach body (Figure $5 \mathrm{~A}-\mathrm{C}$ ), whereas mucous cells were NUCB2-negative (Figure $5 \mathrm{~A}, \mathrm{C}$ ).
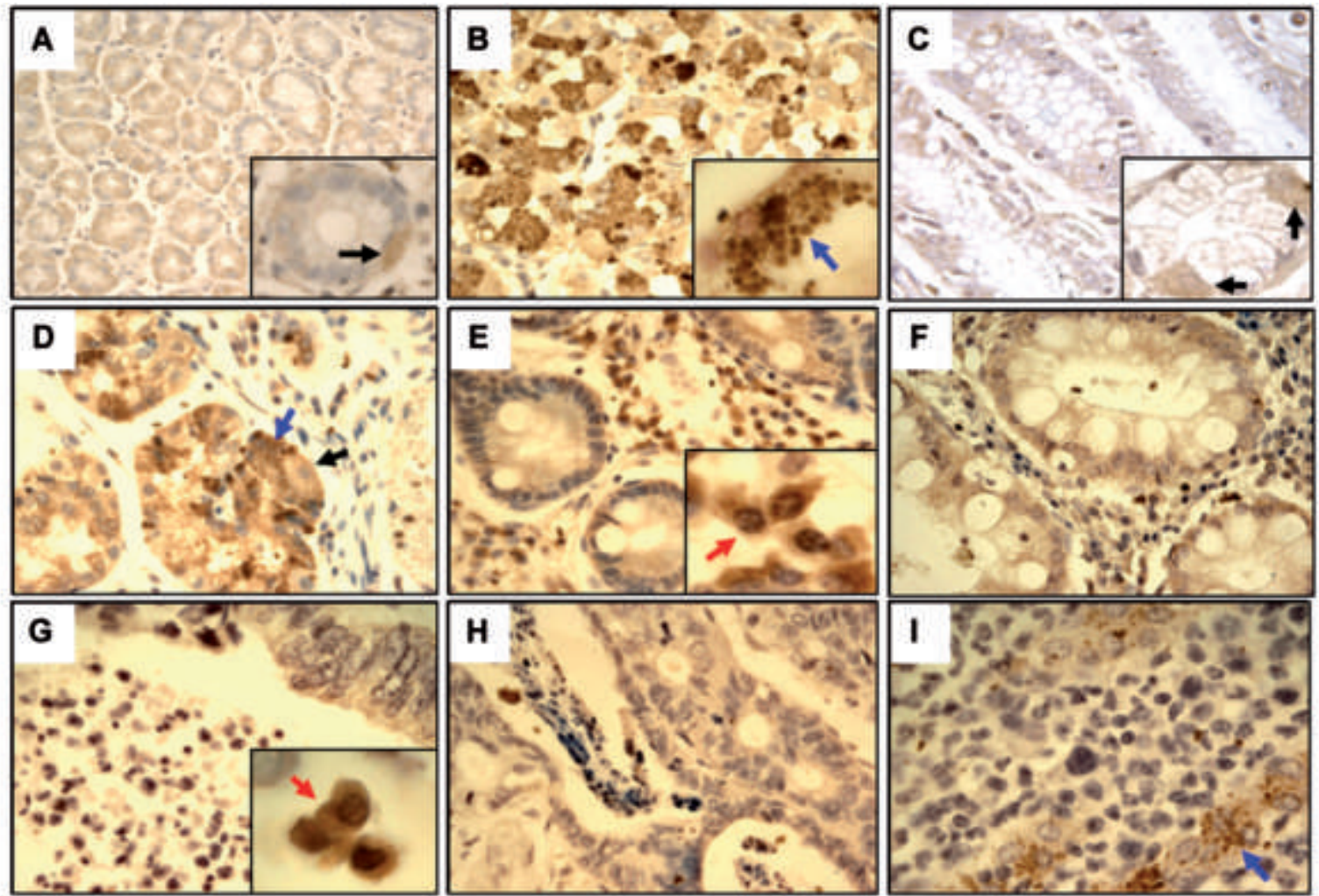

Figure 5. The immunohistochemical analysis of NUCB2 expression in stomach epithelium and gastric lesions. Normal stomach: cardiac glands (A), oxyntic glands (B) and pyloric glands (C). Chronic atrophic gastritis: mild (D), moderate (E) and severe (F). Gastric adenocarcinoma: grade I (G), grade II (H) and grade III (I). Anti-NUCB2 monoclonal antibody was used to stain the tissue sections. NUCB2-speciffic signal was observed in gastric parietal cells (black arrows), chief cells (blue arrows) and plasma cells (red arrows) infiltrated in the gastritis and gastric cancer tissues while mucous cells do not express NUCB2. No NUCB2 positive tumour cells were detected. 
In gastritis, NUCB2 expression was found in the infiltrating plasma cells (Figure $5 \mathrm{E}$ ) as well as in the chief and parietal cells in preserved, presumably functional glandular structures (Figure 5 D) but it was lost in atrophic glands (Figure $5 \mathrm{~F}$ ). Staining of 15 tumour and adjacent normal tissues sections from the same patients whose tissue specimens were used for quantitative RT-PCR and Western blot analyses showed that plasmocytes constituted the main proportion of NUCB2-positive cells in tumour tissues while the tumour cells were NUCB2-negative (Figure 5 G-I). No correlation with the tumour stage or grade was observed.

\section{Antibodies against NUCB2 are not associated with anti-parietal cell antibodies}

Autoantibodies to acid secreting gastric parietal cells (GPC) are involved in the aetiology of autoimmune and atrophic gastritis that are known to be precancerous lesions (Correa 1992). Since NUCB2 expression was localised in gastric parietal cells, we hypothesised that NUCB2 may represent one of the antigens recognised by anti-parietal cell antibodies (APCA). As GPC $\mathrm{H}^{+} / \mathrm{K}^{+}$ATPase is the dominant antigen used to detect the presence of APCA, we expected anti-NUCB2 and anti- $\mathrm{H}^{+} / \mathrm{K}^{+}$ATPase antibodies to coincide in the sera from patients with gastritis and cancer. Four of 13 atrophic gastritis patients' and one of 16 gastric cancer patients' serum samples tested were positive for APCA, however, none of them had anti-NUCB2 antibodies. Similarly, the patients having anti-NUCB2 antibodies were negative for APCA thus demonstrating that induction of anti-NUCB2 response is not associated with the autoimmune processes against GPC.

\section{Discussion}

It has been generally assumed that cancer-associated expression pattern (expression restricted to cancers and testis, the cell type from which the cancer originated, foetal tissues or neurons, overexpression), and a variety of molecular alterations including mutations, translocations and splicing defects may underlie the immunogenicity of SEREX-defined antigens, however, only in a few cases such alterations have been found and demonstrated to induce the immune response (Chen YT 2004; Tureci et al. 1999; Kalnina et al.
2005; Pfreundschuh 2000). Other mechanisms that may render tumour cell proteins immunogenic are altered posttranslational modifications (Canelle et al. 2005; Robinson et al. 2002; Brichory et al. 2001), aberrant subcellular localisation resulting for instance from virus infection (Dong et al. 1994) or altered binding partners of normal self-proteins, or their localisation on the surface of apoptotic tumour cells as shown in the case of $\beta$-actin in medullary breast cancer (Hansen et al. 2001).

In the current study we examined the molecular alterations that may underlie the IgG responses against NUCB2 in gastric cancer patients. The mRNA distribution analysis showed that NUCB2 is expressed in several normal tissues including spleen, thymus and bone marrow. Usually $\mathrm{CD}^{+} \mathrm{T}$ cells recognising antigens that are continuously present in lymphoid tissues are tolerased (Zinkernagel and Hengartner 2001; Bos et al. 2005 ). Since the activation of B cells and Ig class switch to generate IgG class antibodies requires signals from activated $T_{н}$ cells, it is unlikely that the antibodies are directed against the wild-type NUCB2 protein. Furthermore, NUCB2 mRNA and protein levels were found to be downregulated in the majority of gastric tumour tissues when compared to adjacent normal tissues. As the overexpression is supposed to be the most frequent immunogenic stimulus for SEREX-defined antigens, this finding seemed surprising. However, multiple downregulated antigens eliciting cancer-specific immune responses have been identified by SEREX before, including p14.5 in hepatocellular carcinoma (Stenner-Liewen et al. 2000), Seb4B and HDAC5 in colon cancer (Scanlan et al. 2002) and SNT-1/BR-41 in breast cancer (Scanlan et al. 2001). We propose that the immunogenicity of the downregulated antigens is likely to be elicited by the alterations at the DNA, mRNA or protein level resulting in the structural changes of the protein that may give rise to novel epitopes. Hence, some of them may turn out to be tumour suppressors. In fact, the NUCB2 gene is localised to locus 11p15.1-p14, known to be frequently deleted in ovarian and bladder tumours (Kiechle-Schwarz et al. 1994; Shaw and Knowles 1995).

In a previous study we searched for somatic mutations in NUCB2 cDNA by sequencing the complete protein coding region amplified from the tumour tissue of the patient with anti-NUCB2 
antibodies. Although several polymorphisms, including ACA insertion/deletion ( $G$ In 402) were found, none of them turned out to be somatic mutations (Line et al. 2002).

Next, we searched for novel splice variants or transcription initiation sites by applying $5^{\prime} \mathrm{RLM}$ RACE to three gastric cancer specimens that resulted in the identification of an alternative distal promoter and three novel transcript variants differing in the exon composition at the $5^{\prime}$ end of the mRNA, yet none of them could encode for a novel protein isoform. Although the mRNAs transcribed from the newly identified alternative promoter appeared to constitute only a minor fraction of the total NUCB2 mRNA pool, the distal promoter was considerably more active in gastric cancers than in the adjacent normal tissues, particularly in those cases where the total NUCB2 level was strongly downregulated. Hence the usage of the alternative promoter may represent a compensatory mechanism in tumour cells when the transcription from the conventional promoter is shut off. Alternative $5^{\prime}$ UTRs have been shown to play an important role in the post-transcriptional regulation of gene expression by affecting downstream splicing events, translation efficacy, RNA sorting and localisation (Brevini et al. 2007; Smith 2008; Cazzola and Skoda 2000; Tsuda et al. 2000).

In two gastric cancer patient samples, the usage of the distal promoter correlated with the presence of 55 and $57-k \mathrm{Da}$ bands detected by Western blot in gastric tumours but not in adjacent normal tissues. Dephosphorylation experiments demonstrated that the $57 \mathrm{kDa}$ band represents phosphorylated form of the $55 \mathrm{kDa}$ NUCB2 isoform, which by itself is likely to represent novel post-translationally modified isoform. To identify the nature of the modification, we carried out enzymatic deglycosylation, yet this did not affect the mobility of the $52 \mathrm{kDa}$ band in normal tissues and the $55 \mathrm{kDa}$ band in tumours. This treatment should remove most of common 0-linked sugars, however it does not cleave several rare 0 -linked structures such as $\mathrm{N}$-acetylgalactosamine found in mucins or mannose, for example, therefore the glycosylation of NUCB2 can not be definitely ruled out. Although we did not detect any NUCB2-positive cancer cell by immunohistochemistry, the $55 \mathrm{kDa}$ band was detected in AGS gastric cancer cells by Western blotting that implies this isoform is produced by cancer cells not by plasma cells or the remaining functional gastric glands yet at the level undetectable by immunohistochemistry. Alterations in the glycosylation patterns and other post-translational modifications (PTMs) are frequently found in various tumours and have been shown to trigger immune responses to self or tumour proteins (Ohmori and Kanayama 2005; Suriano et al. 2005; Doyle et al. 2006; Doyle and Mamula 2005). However it remains to be determined whether the altered PTM acts as a trigger for the immune response against NUCB2 and whether the transcript variant containing the alternative $5^{\prime}$ UTR is associated with the altered PTM.

Alternatively, the autoantibody production could be elicited by the cleavage of NUCB2 during apoptosis as it has been demonstrated for apoptotic cleavage product of BARDI (Gautier et al. 2000). In fact, NUCB2 was recently shown to be cleaved by caspases and the cleavage sites are located at one of the EF-hand motifs (Figure 1 ) (Valencia et al. 2008).

The identification of multiple functionally unrelated interaction partners and their implication in various processes such as $\mathrm{Ca}^{2+}$ homeostasis (Kroll et al. 1999; Morel-Huaux et al. 2002; Taniguchi et

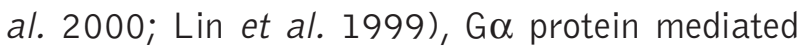
signalling ( $L$ in et al. 2000), unfolded protein response (Tsukumo et al. 2007), TNFRl shedding (Islam et al. 2006) and hypothalamic feeding regulation (Oh et al. 2006; Nonogaki et al. 2008) suggests that nucleobindins are multifunctional proteins having various tissue-specific functions. So far, nothing is known about their function in stomach. The staining of stomach tissue sections with anti-NUCB2 antibody revealed its expression in the chief cells and parietal cells but not in mucous and muscle cells while it is not clear whether it is expressed in G and ECL cells. The main function of chief and parietal cells is the secretion of pepsinogens and gastric acid, respectively. Both secretion processes are coupled and mediated by the intracellular $\mathrm{Ca}^{2+}$ signalling ( $\mathrm{Hou}$ and Schubert 2006; Remy et al. 2007; Kimura et al. 2001; Gritti et al. 2000). The localisation of NUCB2 in the secretory granules of chief cells and its loss in atrophic glands suggests a role in the secretion processes by establishing an agonistreleasable $\mathrm{Ca}^{2+}$ store in ER or Golgi apparatus, signalling via heterotrimeric $G \alpha$ proteins and/or mediating the exocytosis of the secretory granules. 
It also would be of interest to investigate whether the nesfatin 1, the 1-82 aa peptide generated by a proteolytic cleavage of NUCB2 in hypothalamus recently shown to act as a satiety molecule via the serotonin system (Oh et al. 2006; Nonogaki et al. 2008), is also processed and secreted in gastric mucosa as it was shown for other hypothalamic peptides that regulate food intake.

Furthermore, NUCB2 has been shown to have an essential role in the generation of soluble extracellular TNF receptors (TNFRI) through binding to ARTS-1 (aminopeptidase regulator of TNFR1 shedding) extracellular domain in a calciumdependent manner, at least in vascular endothelial cells (Islam et al. 2006). If the same mechanism is operational in the parietal cells, the loss of NUCB2 expression during the progression of gastritis would abolish TNFRI release, thereby rendering the parietal cells more susceptible to TNF$\alpha$ induced apoptosis and thus increasing the severity of atrophy and inflammation.

In summary, we made an attempt to elucidate the molecular mechanism underlying NUCB2 immunogenicity in gastric cancer patients. Novel transcript variants differentially expressed in cancerous tissues and a novel cancer-associated posttranslationally modified NUCB2 isoform were identified, however, no conclusive evidence was gained to prove their association with the immunogenicity of NUCB2. Nevertheless, this study for the first time gained an insight into the physiological functions of NUCB2 in the stomach - it showed that NUCB2 is phosphorylated and expressed in the secretory granules of chief cells and in the cytoplasm of parietal cells in the functioning gastric glands and further we propose the potential role of NUCB2 in the signalling leading to the secretion of gastric acid and pepsinogens. It also would be of interest to investigate whether the loss of NUCB2 is implicated in the progression of inflammation and atrophy mediated by TNF signalling.

\section{Acknowledgements}

This work was supported by grants from Latvian Council of Science (No 05.1623 and 04.1143), EC $6^{\text {th }}$ Framework program ENACT and the fellowships by European Social Fund.

\section{References}

Ballif BA, Mincek NV, Barratt JT, Wilson ML, Simmons DL. Interaction of cyclooxygenases with an apoptosis- and autoimmunity-associated protein. Proc Natl Acad Sci USA 1996;93:5544-9.

Barnikol-Watanabe S, Gross NA, Gotz $H$, Henkel T, Karabinos A, Kratzin $\mathrm{H}$, et al. Human protein NEFA, a novel DNA binding/EFhand/leucine zipper protein. Molecular cloning and sequence analysis of the CDNA, isolation and characterization of the protein. Biol Chem Hoppe Seyler 1994;375:497-512.

Bos $R$, van DS, van $H T$, Kaaijk $P$, Taubert $R$, Kyewski $B$, et al. Expression of a natural tumor antigen by thymic epithelial cells impairs the tumor-protective CD4+ T-cell repertoire. Cancer Res 2005;65:6443-9.

Brevini TA, Cillo F, Antonini S, Tosetti V, Gandolfi F. Temporal and spatial control of gene expression in early embryos of farm animals. Reprod Fertil Dev 2007; 19:35-42.

Brichory FM, Misek DE, Yim AM, Krause MC, Giordano TJ, Beer DG, et al. An immune response manifested by the common occurrence of annexins I and II autoantibodies and high circulating levels of IL-6 in lung cancer. Proc Natl Acad Sci USA 2001;98:9824-9.

Canelle L, Bousquet J, Pionneau C, Deneux L, Imam-Sghiouar N, Caron $\mathrm{M}$, et al. An efficient proteomics-based approach for the screening of autoantibodies. J Immunol Methods 2005;299:77-89.

Cazzola M, Skoda RC. Translational pathophysiology: a novel molecular mechanism of human disease. Blood 2000;95:3280-8.

Chen YT. Identification of human tumor antigens by serological expression cloning: an online review on SEREX. Cancer Immun 2004.

Chen $Y$, Lin P, Qiu S, Peng XX, Looi K, Farquhar MG, et al. Autoantibodies to $\mathrm{Ca} 2+$ binding protein Calnuc is a potential marker in colon cancer detection. Int J Oncol 2007:30:1137-44.

Correa P. Human gastric carcinogenesis: a multistep and multifactorial process--First American Cancer Society Award Lecture on Cancer Epidemiology and Prevention. Cancer Res 1992;52:673540.

Dong X, Hamilton KJ, Satoh M, Wang J, Reeves WH. Initiation of autoimmunity to the $\mathrm{p} 53$ tumor suppressor protein by complexes of p53 and SV40 large T antigen. J Exp Med 1994;179:1243-52.

Doyle HA, Mamula MJ. Posttranslational modifications of self-antigens. Ann N Y Acad Sci 2005;1050:1-9.

Doyle HA, Zhou J, Wolff MJ, Harvey BP, Roman RM, Gee RJ, et al. Isoaspartyl post-translational modification triggers anti-tumor $\mathrm{T}$ and B lymphocyte immunity. J Biol Chem 2006;281:32676-83.

Gautier F, Irminger-Finger I, Gregoire M, Meflah K, Harb J. Identification of an apoptotic cleavage product of BARDI as an autoantigen: a potential factor in the antitumoral response mediated by apoptotic bodies. Cancer Res 2000;60:6895-900.

Gritti I, Banfi G, Roi GS. Pepsinogens: physiology, pharmacology pathophysiology and exercise. Pharmacol Res 2000;41:265-81.

Hansen $\mathrm{MH}$, Nielsen $\mathrm{H}$, Ditzel HJ. The tumor-infiltrating $\mathrm{B}$ cell response in medullary breast cancer is oligoclonal and directed against the autoantigen actin exposed on the surface of apoptotic cancer cells. Proc Natl Acad Sci USA 2001;98:12659-64.

Hou W, Schubert ML. Gastric secretion. Curr Opin Gastroenterol 2006;22:593-8

Islam A, Adamik B, Hawari FI, Ma G, Rouhani FN, Zhang J, et al. Extracellular TNFRI release requires the calcium-dependent formation of a nucleobindin 2-ARTS-1 complex. J Biol Chem 2006;281:6860-73.

Kalnina Z, Zayakin P, Silina K, Line A. 2005. Alterations of pre-mRNA splicing in cancer. Genes Chromosomes Cancer 42:342-57.

Kanai $Y$, Takeda 0 , Miura $K$, Amagai M, Kaneko $T$, Kubota $T$, et al. Induction of autoantibodies in normal mice by injection of nucleobindin and natural occurrence of antibodies against nucleobindin in autoimmune MRL/lpr/lpr mice. Immunol Lett 1995;45:35-42.

Karabinos A, Bhattacharya D, Morys-Wortmann C, Kroll K, Hirschfeld $G$, Kratzin HD, et al. The divergent domains of the NEFA and nucleobindin proteins are derived from an EF-hand ancestor. Mol Biol Evol 1996;13:990-8.

Kiechle-Schwarz M, Bauknecht T, Karck U, Kommoss F, Du BA, Pfleiderer A. Recurrent cytogenetic aberrations and loss of constitutional heterozygosity in ovarian carcinomas. Gynecol Oncol 
1994;55:198-205.

Kimura S, Mieno H, Tamaki K, Inoue M, Chayama K. Nonselective cation channel as a $\mathrm{Ca}(2+)$ influx pathway in pepsinogen-secreting cells of bullfrog esophagus. Am J Physiol Gastrointest Liver Physiol 2001;281:G333-G341.

Kroll KA, Otte S, Hirschfeld G, Barnikol-Watanabe S, Gotz H, Sternbach $\mathrm{H}$ et al. Heterologous overexpression of human NEFA and studies on the two EF-hand calcium-binding sites. Biochem Biophys Res Commun 1999;260:1-8.

Kubota T, Watanabe N, Kaneko T, Satake F, Miura K, Kurosawa Y, et al. Activation of autoreactive $T$ cells that help nucleobindin-injected mice produce anti-DNA antibodies. Immunol Lett 2001;75:111-5.

Lavoie C, Meerloo T, Lin P, Farquhar MG. Calnuc, an EF-hand Ca(2+)binding protein, is stored and processed in the Golgi and secreted by the constitutive-like pathway in AtT20 cells. Mol Endocrinol 2002; 16:2462-74.

Lin $\mathrm{P}$, Fischer T, Weiss T, Farquhar MG. Calnuc, an EF-hand $\mathrm{Ca}(2+)$ binding protein, specifically interacts with the C-terminal alpha5helix of G(alpha)i3. Proc Natl Acad Sci USA 2000;97:674-9.

Lin P, Yao Y, Hofmeister R, Tsien RY, Farquhar MG. Overexpression of CALNUC (nucleobindin) increases agonist and thapsigargin releasable Ca2+ storage in the Golgi. J Cell Biol 1999;145:279-89.

Line A, Stengrevics A, Slucka Z, Li G, Jankevics E, Rees RC. Serological identification and expression analysis of gastric cancerassociated genes. Br J Cancer 2002;86:1824-30.

Miura K, Titani K, Kurosawa Y, Kanai Y. Molecular cloning of nucleobindin, a novel DNA-binding protein that contains both a signal peptide and a leucine zipper structure. Biochem Biophys Res Commun 1992;187:375-80.

Morel-Huaux VM, Pypaert M, Wouters S, Tartakoff AM, Jurgan U, Gevaert K, et al. The calcium-binding protein p54/NEFA is a novel luminal resident of medial Golgi cisternae that traffics independently of mannosidase II. Eur J Cell Biol 2002;81:87-100.

Nonogaki K, Ohba Y, Sumii M, Oka Y. Serotonin systems upregulate the expression of hypothalamic NUCB2 via $5-\mathrm{HT} 2 \mathrm{C}$ receptors and induce anorexia via a leptin-independent pathway in mice. Biochem Biophys Res Commun 2008;372:186-90.

Oh I, Shimizu H, Satoh T, Okada S, Adachi S, Inoue K, et al. Nature 2006;443:709-712.

Ohmori $\mathrm{H}$, Kanayama N. Immunogenicity of an inflammation-associated product, tyrosine nitrated self-proteins. Autoimmun Rev 2005; 4:224-9.

Pfreundschuh M. Exploitation of the B cell repertoire for the identification of human tumor antigens. Cancer Chemother Pharmacol 2000;46 Suppl:S3-S7.

Remy C, Kirchhoff P, Hafner P, Busque SM, Mueller MK, Geibel JP, Wagner CA. 2007. Stimulatory pathways of the Calcium-sensing receptor on acid secretion in freshly isolated human gastric glands.
Cell Physiol Biochem 19:33-42.

Robinson WH, DiGennaro C, Hueber W, Haab BB, Kamachi M, Dean EJ, et al. Autoantigen microarrays for multiplex characterization of autoantibody responses. Nat Med 2002;8:295-301.

Sahin U, Tureci 0, Pfreundschuh M. Serological identification of human tumor antigens. Curr Opin Immunol 1997;9:709-16.

Scanlan MJ, Gout I, Gordon CM, Williamson B, Stockert E, Gure A0, et al. Humoral immunity to human breast cancer: antigen definition and quantitative analysis of mRNA expression. Cancer Immun $2001 ; 1: 4$.

Scanlan MJ, Welt S, Gordon CM, Chen YT, Gure A0, Stockert E, et al. Cancer-related serological recognition of human colon cancer: identification of potential diagnostic and immunotherapeutic targets. Cancer Res 2002;62:4041-7.

Shaw ME, Knowles MA. Deletion mapping of chromosome 11 in carcinoma of the bladder. Genes Chromosomes Cancer 1995;13:1-8.

Smith L. Post-transcriptional regulation of gene expression by alternative 5 -untranslated regions in carcinogenesis. Biochem Soc Trans 2008;36:708-11.

Stenner-Liewen F, Luo G, Sahin U, Tureci 0, Koslovski M, Kautz I, et al. Definition of tumor-associated antigens in hepatocellular carcinoma. Cancer Epidemiol Biomarkers Prev 2000;9:285-90.

Suriano R, Ghosh SK, Ashok BT, Mittelman A, Chen Y, Banerjee A, Tiwari RK. Differences in glycosylation patterns of heat shock protein, gp96: implications for prostate cancer prevention. Cancer Res 2005;65:6466-75.

Taniguchi N, Taniura H, Niinobe M, Takayama C, Tominaga-Yoshino K, Ogura $A$, et al. The postmitotic growth suppressor necdin interacts with a calcium-binding protein (NEFA) in neuronal cytoplasm. J Biol Chem 2000;275:31674-81.

Tsuda M, Egashira M, Niikawa N, Wada Y, Honke K. Cancer-associated alternative usage of multiple promoters of human GalCer sulfotransferase gene. Eur J Biochem 2000;267:2672-9.

Tsukumo Y, Tomida A, Kitahara 0, Nakamura Y, Asada S, Mori K, et al. Nucleobindin 1 controls the unfolded protein response by inhibiting ATF6 activation. J Biol Chem 2007;282:29264-72.

Tureci 0, Sahin U, Zwick C, Neumann F, Pfreundschuh M. Exploitation of the antibody repertoire of cancer patients for the identification of human tumor antigens. Hybridoma 1999;18:23-8.

Valencia CA, Cotten SW, Duan J, Liu R. Modulation of nucleobindin-1 and nucleobindin-2 by caspases. FEBS Lett 2008;582:286-290.

Wendel M, Sommarin Y, Bergman T, Heinegard D. Isolation, characterization, and primary structure of a calcium-binding $63-\mathrm{kDa}$ bone protein. J Biol Chem 1995;270:6125-33.

Zinkernagel RM, Hengartner $\mathrm{H}$. Regulation of the immune response by antigen. Science $2001 ; 293: 251-3$. 
Z. Kalnina et al. 